\title{
Fatores relacionados ao trauma perineal no parto normal em nulíparas*
}

\author{
FACTORS RELATED TO PERINEAL TRAUMA IN NORMAL BIRTHS IN NULLIPAROUS
}

FACTORES RELACIONADOS AL TRAUMA PERINEAL EN EL PARTO NORMAL EN NULÍPARAS

\section{Leila Barreto Scarabotto', Maria Luiza Gonzalez Riesco²}

\section{RESUMO}

Inúmeros estudos têm sido realizados com a finalidade de contribuir para a prevenção do trauma perineal no parto normal. O objetivo do presente estudo foi relacionar a altura do períneo, duração do período expulsivo, variedade de posição no desprendimento cefálico, tipo de puxo, presença de circular de cordão, peso do recém-nascido e ardor na vulva ao urinar com a ocorrência de lacerações perineais. A pesquisa foi realizada em 2003, no Centro de Parto Normal do Amparo Maternal, com uma amostra de 67 parturientes sem partos vaginais anteriores. Os resultados mostraram que não houve diferença estatisticamente significante em relação às variáveis analisadas.

\section{DESCRITORES}

Parto normal.

Períneo (lesões).

Enfermagem obstétrica.

\begin{abstract}
Many studies have been undertaken with the purpose of contributing towards the prevention of perineal trauma in normal birth. The objective of this study was to relate height of the perineum, duration of the second stage of labor, variation of the position of the head detaching, kind of effort, presence of the umbilical cord around the babies' neck, birth weight and vulva's ardor to urinate with the occurrence of perineal laceration. The study was undertaken in 2003 at the Normal Birth Center of the Amparo Maternal, with a sample consisting of 67 women in labor without previous vaginal births. The results demonstrated that there were no significant statistical differences between the variables verified.
\end{abstract}

\section{KEY WORDS}

Natural childbirth.

Perineum (injuries).

Obstetrical nursing.

\section{RESUMEN}

Innumerables estudios han sido realizados con la finalidad de contribuir en la prevención del trauma perineal, en el parto normal. El objetivo del presente estudio fue relacionar la altura del perineo, duración del período expulsivo, variedad de posición en el desprendimiento cefálico, tipo de pujo, presencia de circular de cordón, peso del recién nacido y ardor en la vulva al orinar con la ocurrencia de laceraciones perineales. La investigación fue realizada en el 2003, en el Centro de Parto Normal do Amparo Maternal, con una muestra de 67 parturientas sin partos vaginales anteriores. Los resultados mostraron que no hubo diferencia estadísticamente significativa en relación a las variables analizadas.

\section{DESCRIPTORES}

Parto normal.

Perineo (lesiones).

Enfermería obstétrica.

\footnotetext{
* Extraído da Dissertação "Uso da enzima hialuronidase na prevenção de lacerações perineais no parto normal", Escola de normal", Escola de Universidade de São Paulo (EEUSP), 2004.

1 Enfermeira Obsté-

trica. Mestre em Enfermagem. Obstetriz do Centro de Parto Normal do Amparo Maternal. Membro do Grupo de Pesquisa

"Enfermagem e

Assistência ao Parto: Modelos, Agentes e Práticas" leila@fasb.com.br 2 Enfermeira Obstétrica. Professora Doutora do Departamento de Enfermagem MaternoInfantil e Psiquiátrica da EEUSP. Coordenadora do Grupo de Pesquisa "Enfermagem e Assistência ao Parto: Modelos, Agentes e Práticas". riesco@usp.br
} 


\section{INTRODUÇÃO}

A maioria das mulheres sofre algum tipo de trauma perineal no parto normal, em razão de lacerações perineais espontâneas ou de episiotomia ${ }^{(1-2)}$. As lacerações espontâneas são classificadas como primeiro grau (afetam pele e mucosa), segundo grau (estendem-se até os músculos perineais) ou terceiro grau (atingem o músculo esfíncter do ânus). No caso da episiotomia, além da pele e da mucosa, são habitualmente seccionados os músculos transverso superficial do períneo e bulbocarvernoso ${ }^{(3)}$.

No parto normal, a ocorrência de lacerações perineais depende de diversos fatores, que podem estar relacionados às condições maternas, ao feto, ao parto em si e à própria episiotomia, amplamente utilizada para evitar lacerações na região, que constitui um trauma perineal, por vezes, mais severo que as lacerações espontâneas.

Entre 1915 e 1925, a episiotomia era usada de forma profilática para evitar traumas perineais, prevenir a morbidade e mortalidade infantil e problemas ginecológicos, como: cistocele, retocele e relaxamento do assoalho pélvico ${ }^{(4)}$.

A episiotomia tende a ser abandonada como rotina e, em alguns países, índices menores que 20\% têm sido alcançados; a fibrose por episiotomia anterior é uma das principais indicações para sua manutenção ${ }^{(5)}$. Além de não haver evidências confiáveis de melhores resultados maternos ou perinatais com emprego rotineiro da episiotomia, existem evidências claras de que seu uso comporta riscos ${ }^{(6)}$.

A revisão sistemática de seis ensaios controlados randomizados, realizados entre 1984 e 1994, mostrou que as vantagens do uso restrito da episiotomia são visíveis, com menor incidência de trauma perineal posterior, necessidade de suturas, referência para dor e traumas vaginais severos e complicações, embora exista um risco maior de laceração perineal anterior ${ }^{(7)}$.

Estudo realizado entre janeiro de 1988 e dezembro de 1991, no Hospital Universitário de Leiden, Holanda, com 1.272 primíparas que tiveram partos vaginais, verificou os fatores que poderiam influenciar a decisão para realização ou não da episiotomia, demonstrando dependência maior da educação do profissional do que de fatores médicos ${ }^{(8)}$.

Outra revisão sistemática sobre o uso da episiotomia em seis trabalhos clínicos aleatorizados com 6.600 mulheres, comparando a episiotomia seletiva com a de rotina, revelou os seguintes resultados: redução de $20 \%$ em trauma perineal severo, $12 \%$ em trauma perineal posterior, $29 \%$ de dor perineal na alta e $71 \%$ nas complicações de cicatrização, com a episiotomia seletiva ${ }^{(9)}$.

Conforme alguns estudos ${ }^{(1,10-11)}$, as indicações para realização de episiotomia são: sofrimento fetal; feto gran- de; prematuridade; distensibilidade restrita do assoalho pélvico que engloba primiparidade precoce e tardia; distância entre a fúrcula e o ânus menor que três centímetros e edema de vulva (para evitar laceração denteada); progressão insuficiente do parto; ameaça de laceração de terceiro grau; apresentação pélvica e distocia de ombro.

$\mathrm{Na}$ Irlanda, em 1982, foi realizado estudo que comparou mulheres com e sem episiotomia, porém com lacerações espontâneas de segundo grau, mostrando parâmetros na medição de dor idênticos e uma proporção similar de mulheres sem dor do primeiro ao quarto dia depois do parto ${ }^{(12)}$.

Em outro estudo randomizado controlado com 61 puérperas, no quarto dia após o parto, $65,5 \%$ das mulheres com episiotomia referiam dor espontânea contra $41,4 \%$ com rotura de segundo grau, havendo associação estatisticamente significante entre dor perineal e episiotomia ${ }^{(13)}$.

Na Argentina, entre 1990 e 1992, estudo clínico que envolveu 2.606 mulheres, mostrou que a dor perineal e as complicações locais estavam associadas à ocorrência de episiotomia ${ }^{(14)}$.

Por sua vez, o ardor da vulva ao urinar é um sintoma no pós-parto citado por algumas mulheres, sem destaque na literatura que discute a morbidade associada ao trauma perineal no parto normal.

As lesões genitais no parto vaginal podem ser prevenidas pela boa assistência, o que inclui mudança de condutas, como restrição do uso de episiotomia, de ocitocina e da posição horizontal. Afirma, ainda, que o tônus da musculatura da vagina depende mais do exercício e da consciência da mulher, de sua contração e relaxamento do que de cirurgias de rotina ${ }^{(5)}$.

Em estudos relacionados à opção da mulher pela posição no parto, evitando a posição litotômica, foi encontrada prevalência de períneo íntegro no parto em posição lateral esquerda, com diferença estatisticamente significante em comparação com a posição litotômica associada ao aumento de lacerações no períneo ${ }^{(10,15-16)}$.

Ainda como fatores relacionados ao parto ou ao feto, que podem estar associados às lacerações perineais, vale destacar a duração do período expulsivo, a variedade de posição no desprendimento do pólo cefálico, o tipo de puxo realizado pela parturiente, a presença de circular de cordão umbilical e o peso do recém-nascido.

Com relação à duração do segundo estágio do parto, há estudos que consideram não haver um limite fixo, e o tempo dessa fase pode diminuir efetivamente com a episiotomia, entre um e nove minutos. No entanto, esses minutos a menos não representam uma resposta clara para justificar a realização da episiotomia de rotina ${ }^{(11-12)}$. 
A Organização Mundial de Saúde ${ }^{(1)}$ recomenda esperar até que a própria mulher sinta necessidade de realizar o puxo, permitindo que ela responda aos sinais e sintomas de seu corpo com esforços expulsivos mais curtos, movimentos respiratórios superficiais e rápidos e intervalos para descanso.

Estudo anteriormente referido ${ }^{(16)}$ encontrou a freqüência de 95,7\% de expulsão em variedade occipto-púbica, do total de 70 partos. No mesmo estudo, a circular de cordão ocorreu em 35,7\% dos casos, e as manobras de redução e de clampeamento do cordão foram usadas para desfazê-la, sem associação significante com a ocorrência de lacerações perineais. A respeito da relação entre lacerações e peso do RN, dentre os cinco partos com peso fetal maior ou igual a 3.500 gramas ocorreu apenas um caso de laceração de segundo grau, sem traumas mais severos ${ }^{(16)}$.

\section{OBJETIVO}

Relacionar a ocorrência de lacerações perineais no parto normal com a altura do períneo, duração do período expulsivo, variedade de posição no desprendimento cefálico, tipo de puxo, presença de circular de cordão, peso do recém-nascido e ardor na vulva ao urinar.

\section{METODOLOGIA}

Estudo transversal sobre fatores relacionados ao trauma perineal no parto normal, em parturientes que atenderam os seguintes critérios: ser nulípara ou, mais precisamente, não ter parto vaginal anterior; ter gestação única, a termo, com feto vivo em apresentação cefálica fletida; não ter recebido infusão endovenosa de ocitocina; não ter sido submetida a preparo do períneo durante a gestação e ter o parto na posição lateral esquerda.

A pesquisa foi realizada entre os meses de novembro de 2002 a maio de 2003, no Centro de Parto Normal do Amparo Maternal (CPN-AM), após aprovação pelo Comitê de Ética em Pesquisa do Hospital Universitário da Universidade de São Paulo.

O tamanho da amostra foi determinado pela estimativa de lacerações perineais nos partos ocorridos no CPN-AM, com proporção que corresponde à suposição de 50\% de lacerações, nos casos sem episiotomia ${ }^{(17)}$. Para a obtenção de um intervalo de confiança de $95 \%$, foi definida uma amostra de 67 mulheres.

Os dados foram coletados com uso de um formulário com identificação da parturiente e informações relativas ao parto, ao recém-nascido e às condições do períneo na alta da puérpera. Os partos foram assistidos por uma das pesquisadoras, com padronização da técnica de proteção perineal no período expulsivo. Foi feito o agrupamento das mulheres do estudo segundo o desfecho obtido em relação às condições do períneo no parto, considerando os grupo com e sem laceração. No grupo com laceração foram incluídas as 51 mulheres que apresentaram lacerações perineais de primeiro e segundo graus. No grupo sem laceração foram incluídas as 16 mulheres que apresentaram o períneo íntegro ou com solução de continuidade que não exigiu sutura. Quanto ao grau da laceração, $42(82,4 \%)$ foram de primeiro e $9(17,6 \%)$, de segundo.

A análise dos dados foi realizada a partir do banco de dados do aplicativo Excel, calculando-se as freqüências absolutas e relativas para variáveis qualitativas e as medidas de tendência central e dispersão para as variáveis quantitativas. A análise comparativa foi estabelecida pelos testes Qui-Quadrado, Exato de Fisher e não-paramétrico de Mann-Whitney, realizados na forma bi-caudal, considerando-se estatisticamente significantes os valores de $p$ inferiores a 0,05 .

\section{RESULTADOS}

Os dados mostraram que a maioria das mulheres $(80,6 \%)$ tinha menos de 25 anos, com média de 21 anos, desvio padrão de 4,5, sendo a idade mínima e máxima de 15 e 36 anos, respectivamente. A proporção de adolescentes foi de 44,8\%. Com relação à cor, 53,7\% eram brancas e 46,3\% não brancas.

As Tabelas 1 e 2 apresentam a relação entre altura do períneo e duração do período expulsivo e a ocorrência e grau de lacerações perineais; os dados apontam que não houve diferença estatisticamente significante entre essas variáveis.

Em relação à variedade de posição no desprendimento do pólo cefálico, em $100 \%$ dos casos, ocorreram em occipto-púbica.

Tabela 1 - Valores de média, desvio-padrão, mínimo e máximo da altura do períneo, em centímetros, e da duração do período expulsivo, em minutos, segundo a condição do períneo - São Paulo - 2003 (n=67)

\begin{tabular}{l|c|c|c|c|c|c|c}
\hline Variável & $\mathbf{n}$ & $\overline{\mathbf{x}}$ & $\mathbf{d} . \mathbf{p}$. & Med. & Mín. & Máx. & $\mathbf{p}$-valor \\
\hline Altura do períneo (cm) & & & & & & & \\
Com laceração & 51 & 2,9 & 0,3 & 3,0 & 2,5 & 3,5 & ${ }^{(1)} 0,809$ \\
Sem laceração & 16 & 2,8 & 0,4 & 3,0 & 2,5 & 3,5 & \\
Duração do período expulsivo (min) & 51 & 29,0 & 10,0 & 30,0 & 15,0 & 60,0 & ${ }^{(1)} 0,119$ \\
Com laceração & 16 & 32,4 & 9,1 & 30,5 & 15,0 & 49,0 & \\
Sem laceração & &
\end{tabular}

(1) Teste de Mann-Whitney 
Tabela 2 - Valores de média, desvio-padrão, mínimo e máximo da altura do períneo, em centímetros, e da duração do período expulsivo, em minutos, segundo o grau de laceração perineal - São Paulo - 2003 (n=51)

\begin{tabular}{l|r|r|r|r|r|c|c}
\hline Variável & $\mathbf{n}$ & $\overline{\mathbf{x}}$ & $\mathbf{d . p .}$ & Med. & Mín. & Máx. & $\mathbf{p}$-valor \\
\hline Altura do períneo (cm) & & & & & & & \\
Primeiro grau & 42 & 2,8 & 0,3 & 3,0 & 2,5 & 3,0 & $(1) 0,221$ \\
Segundo grau & 9 & 2,9 & 0,2 & 3,0 & 2,5 & 3,0 & \\
Duração do período expulsivo (min) & 42 & 28,7 & 10,3 & 29,0 & 15,0 & 60,0 & $(1) 0,486$ \\
Primeiro grau & 9 & 30,4 & 9,1 & 30,0 & 20,0 & 45,0 & \\
Segundo grau & &
\end{tabular}

(1) Teste de Mann-Whitney

A associação entre tipo de puxo realizado pela parturiente e ocorrência e grau de lacerações perineais mostra que houve distribuição homogênea de casos de laceração e de integridade perineal, e de lacerações de primeiro e segundo graus, considerando os puxos exclusivamente espontâneos e as situações em que houve o direcionamento do puxo (Tabelas 3 e 4 ).

As Tabelas 3 e 4 indicam a distribuição homogênea da freqüência de lacerações e de integridade perineal e graus de laceração, na presença de circular de cordão, sem associação estatisticamente significante. A manobra de redução foi utilizada em $100 \%$ dos casos para desfazer a circular.

Pode-se considerar, no entanto, que os resultados apontam uma tendência à laceração mais profunda com uso de puxos dirigidos e na presença de circular de cordão umbilical (Tabela 4).

Tabela 3 - Condição do períneo segundo o tipo de puxo, presença de circular de cordão e peso do recém-nascido São Paulo - 2003

\begin{tabular}{|c|c|c|c|c|c|c|c|}
\hline \multirow{3}{*}{ Variável } & \multicolumn{4}{|c|}{ Condição do períneo } & & & \multirow{3}{*}{ p-valor } \\
\hline & \multicolumn{2}{|c|}{ Com laceração } & \multicolumn{2}{|c|}{ Sem laceração } & \multicolumn{2}{|c|}{ Total } & \\
\hline & $n=51$ & $\%$ & $\mathrm{n}=16$ & $\%$ & $\mathrm{n}=67$ & $\%$ & \\
\hline \multicolumn{8}{|l|}{ Puxo } \\
\hline Espontâneos & 44 & 75,9 & 14 & 24,1 & 58 & 100 & \multirow{2}{*}{ (1) 1,000} \\
\hline Espontâneos e dirigidos & 7 & 77,8 & 2 & 22,2 & 9 & 100 & \\
\hline \multicolumn{8}{|l|}{ Circular de cordão } \\
\hline Sim & 12 & 85,7 & 2 & 14,3 & 14 & 100 & \multirow{2}{*}{ (1) 0,490} \\
\hline Não & 39 & 73,6 & 14 & 26,4 & 53 & 100 & \\
\hline \multicolumn{8}{|l|}{ Peso do RN (g) } \\
\hline $2000 \vdash 2500$ & 2 & 66,7 & 1 & 33,3 & 3 & 100 & \multirow{4}{*}{ (1) 0,305} \\
\hline $2500+3000$ & 9 & 60,0 & 6 & 40,0 & 15 & 100 & \\
\hline $3000+3500$ & 31 & 79,5 & 8 & 20,5 & 39 & 100 & \\
\hline$\geqslant 3500$ & 9 & 90,0 & 1 & 10,0 & 10 & 100 & \\
\hline
\end{tabular}

(1) Teste Exato de Fisher

Tabela 4 - Grau de laceração perineal segundo o tipo de puxo, presença de circular de cordão e peso do recém-nascido - São Paulo - 2003

\begin{tabular}{|c|c|c|c|c|c|c|c|}
\hline \multirow{3}{*}{ Variável } & \multicolumn{4}{|c|}{ Condição do períneo } & & & \multirow{3}{*}{ p-valor } \\
\hline & \multicolumn{2}{|c|}{ Primeiro grau } & \multicolumn{2}{|c|}{ Segundo grau } & \multicolumn{2}{|c|}{ Total } & \\
\hline & $n=42$ & & $n=9$ & $\%$ & $\mathrm{n}=51$ & $\%$ & \\
\hline \multicolumn{8}{|l|}{ Puxo } \\
\hline Espontâneos & 38 & 86,4 & 6 & 13,6 & 44 & 100 & \multirow{2}{*}{ (1) 0,095} \\
\hline Espontâneos e dirigidos & 4 & 57,1 & 3 & 42,9 & 7 & 100 & \\
\hline \multicolumn{8}{|l|}{ Circular de cordão } \\
\hline Sim & 9 & 75,0 & 3 & 25,0 & 12 & 100 & \multirow{2}{*}{ (1) 0,424} \\
\hline Não & 33 & 84,6 & 6 & 15,4 & 39 & 100 & \\
\hline \multicolumn{8}{|l|}{ Peso do RN (g) } \\
\hline $2000+2500$ & 2 & 100 & - & - & 2 & 100 & \multirow{4}{*}{ (1) 0,442} \\
\hline $2500 \vdash 3000$ & 9 & 100 & - & - & 9 & 100 & \\
\hline $3000 \vdash 3500$ & 24 & 77,4 & 7 & 22,6 & 31 & 100 & \\
\hline$\geqslant 3500$ & 7 & 77,8 & 2 & 22,2 & 9 & 100 & \\
\hline
\end{tabular}

(1) Teste Exato de Fisher 
Quanto ao peso do recém-nascido, embora também não exista diferença estatisticamente significante, houve uma concentração maior de recém-nascidos com peso maior ou igual a 3.000 gramas, entre as parturientes com laceração perineal (Tabelas 3 e 4). A média de peso dos recém-nascidos foi de 3.159,9 gramas, sendo que nas nove parturientes com laceração perineal de segundo grau a média de peso dos bebês foi de 3.264,4 gramas. O menor valor do Apgar foi 8 e 9 , no primeiro e quinto minutos, respectivamente.

Observa-se que a maioria das mulheres que tiveram laceração perineal apresentaram ardor na vulva ao urinar, porém os resultados não mostraram diferença estatisticamente significante em relação ao grupo com períneo íntegro (Tabela 5).

Tabela 5 - Ardor na vulva ao urinar, segundo a condição do períneo - São Paulo - 2003

\begin{tabular}{|c|c|c|c|c|c|c|c|}
\hline \multirow{3}{*}{ Ardor na vulva } & \multicolumn{4}{|c|}{ Condição do períneo } & & & \multirow{3}{*}{ p-valor } \\
\hline & \multicolumn{2}{|c|}{ Com laceração } & \multicolumn{2}{|c|}{ Sem laceração } & \multicolumn{2}{|c|}{ Total } & \\
\hline & $\mathbf{n}$ & $\%$ & $\mathbf{n}$ & $\%$ & $\mathrm{~N}$ & $\%$ & \\
\hline Sim & 34 & 66,7 & 8 & 50,0 & 42 & 62,7 & \\
\hline Não & 17 & 33,3 & 8 & 50,0 & 25 & 37,3 & (1) 0,229 \\
\hline Total & 51 & 100 & 16 & 100 & 67 & 100 & \\
\hline
\end{tabular}

(1) Teste Qui-Quadrado

\section{DISCUSSÃO}

Os estudos confirmam a falta de evidências científicas para associar o trauma perineal à idade materna. Resultados semelhantes estão em diversos trabalhos ${ }^{(10,18-20)}$, com destaque para a ausência de significância na associação entre trauma perineal e idade materna. No entanto, o estudo com 1.809 primíparas que tiveram parto vaginal no Hospital da Universidade de Michigan, entre 1996 e 1998, verificou um incremento de $6 \%$ na probabilidade de ocorrer laceração perineal para cada ano a mais na idade ${ }^{(21)}$.

A idade das mulheres do presente estudo, com média de 21 anos e menos da metade das parturientes adolescentes, é superior àquela obtida no estudo ${ }^{(16)}$ que encontrou a média de idade de 19 anos (d.p. 3,0) e 60,0\% das mulheres com menos de 20 anos, porém, sem estabelecer relação entre a idade materna e a ocorrência de trauma perineal.

Quanto à cor das gestantes, houve prevalência de mulheres brancas, destacando-se dois estudos em que os autores afirmam que houve menos casos de laceração de segundo grau e mais partos com períneo íntegro em negras ${ }^{(21-22)}$.

Uma das indicações para a realização de episiotomia é a distensibilidade restrita do assoalho pélvico, com altura do períneo menor que três centímetros. A distância entre a fúrcula e o orifício do ânus poderia estar associada ao trauma perineal, considerando que uma altura menor favorece lacerações de terceiro grau e uma altura exagerada reduziria a distensibilidade nessa região ${ }^{(1-10)}$. Entre as mulheres deste estudo, essa variável não mostrou associação estatisticamente significante com a ocorrência e grau de lacerações perineais $(\mathrm{p}=0,809 \mathrm{e} \mathrm{p}=0,221$, respectivamente).

Ainda, quanto à distensibilidade do períneo, vale destacar que o estudo não incluiu parturientes que realizaram algum preparo do períneo durante a gestação. Embora sejam escassos os trabalhos associando o trauma perineal ao preparo do períneo, a massagem perineal na gestação e no parto pode contribuir na prevenção de lacerações de segundo e terceiro graus, episiotomia e partos instrumentais ${ }^{(23)}$. Os autores verificaram esse benefício em estudo randomizado prospectivo com 861 nulíparas, entre 1994 e 1995, na Inglaterra.

Nesta pesquisa, outros fatores foram controlados, como o uso de ocitocina, a posição no parto e a apresentação fetal. Em serviços com uso restrito de episiotomia, a apresentação pélvica constitui uma das indicações para o procedimento $^{(13)}$.

A infusão intravenosa de ocitocina pode estar associada a distocias ou, simplesmente, ser utilizada para acelerar o parto, com aumento das contrações e da pressão intra-uterina no estágio expulsivo do parto. Como conseqüência, pode ocorrer o desprendimento cefálico abrupto, com laceração perineal.

Outro fator associado à ocorrência de trauma perineal é a posição adotada no parto. Pesquisas mostram que o parto na posição lateral esquerda reduz significativamente o uso da episiotomia, além do risco de lacerações severas, pelo fato de diminuir a tensão perineal durante a passagem do feto $^{(10,24)}$.

No presente estudo, a duração do período expulsivo manteve-se nos limites aceitáveis para nulíparas, de até duas horas, segundo o Ministério da Saúde ${ }^{(2)}$, sem associação estatisticamente significante com a freqüência e severidade das lacerações ( $p=0,119$ e $p=0,486$, respectivamente). Vários trabalhos apresentam resultados semelhantes, com relação à média do período expulsivo ${ }^{(25-26)}$. 
No estudo anteriormente citado $^{(16)}$, observou-se uma grande variabilidade na duração do período expulsivo, com o mínimo de um e máximo de 72 minutos, sem diferença estatisticamente significante com relação à técnica utilizada na proteção perineal e no manejo do feto. No entanto, a precisão desta variável pode ser difícil de estabelecer pela indefinição do início exato da expulsão, tendo-se considerado, para este trabalho, a dilatação total da cérvix uterina com o pólo cefálico em +2 no plano de De Lee.

Vale considerar que o desprendimento cefálico ocorreu em variedade occipto-púbica nas 67 parturientes da pesquisa, excluindo-se sua relação com a maior duração da expulsão ou a ocorrência de lacerações perineais.

Os esforços de expulsão involuntários estão ligados ao progresso adequado do parto e à boa vitalidade e que o puxo espontâneo está associado ao índice de Apgar no primeiro minuto acima de 8 para todos os recém-nascidos ${ }^{(26)}$. Outro trabalho também indica que, embora as evidências com relação ao tipo de puxo mais adequado no parto sejam insuficientes, os puxos prolongados e precoces resultam em uma pequena redução da duração do segundo estágio de parto e isto não parece trazer qualquer benefício, mas sim, um maior comprometimento das trocas gasosas materno-fetais ${ }^{(27)}$.

Estudo $^{(19)}$ mostrou associação estatisticamente significante entre o rompimento de tecidos e o puxo dirigido, com maior integridade perineal e menor grau de laceração no períneo com o puxo espontâneo, considerando que $28 \%$ das mulheres usaram o puxo espontâneo e $72 \%$ o dirigido.

Embora o puxo exclusivamente espontâneo tenha foi realizado pela maioria das parturientes deste estudo, os resultados perineais foram semelhantes em relação à freqüência de lacerações $(p=1,000)$. No entanto, mesmo sem significância estatística ( $\mathrm{p}=0,095)$, houve mais lacerações de segundo grau entre as parturientes que realizaram o puxo espontâneo e dirigido, em relação àquelas que utilizaram apenas o puxo espontâneo (42,9 contra 13,6\%).

Quanto à circular de cordão, esta esteve presente em 20,9\% dos casos, utilizando-se a manobra de redução para desfazê-la em $100 \%$ deles. Neste estudo, a presença da circular não esteve associada à maior ocorrência de lacerações perineais $(\mathrm{p}=0,210)$, coincidindo com o resultado obtido em pesquisa $^{(10)}$, que teve a ocorrência de $21,2 \%$ de circular, sem associação com maior frequiência de trauma perineal. O grau da laceração manteve-se independente da presença de circular $(\mathrm{p}=0,424)$, porém a proporção de laceração de segundo grau foi ligeiramente superior quando o feto apresentava circular de cordão umbilical (25,0 contra 15,4\%).

Com relação ao peso do recém-nascido, a média foi de $3.159,9$ gramas e a maior proporção de lacerações perineais concentrou-se nos partos com recém-nascidos entre 3.000 e 3.495 gramas, seguida daqueles com peso maior ou igual a 3.500 gramas, sem associação estatisticamente significante entre o peso fetal e a ocorrência o grau de lacerações perineais ( $\mathrm{p}=0,305$ e $\mathrm{p}=0,442$, respectivamente). Vale destacar que a média do peso dos bebês nos nove partos em que ocorreu laceração de segundo grau foi 3.264,4 gramas.

No estudo de coorte randomizado ${ }^{(28)} \mathrm{com} 1.211$ mulheres com parto normal atendidas no Hospital da Universidade do Novo México, o peso fetal esteve associado à maior freqüência de trauma perineal e os partos com fetos acima de 3.500 gramas, associados à realização da episiotomia. Outro estudo aponta que, para cada 500 gramas de aumento no peso fetal, a parturiente tem $30 \%$ a mais de probabilidade de apresentar laceração perineal ${ }^{(21)}$.

Vale considerar que a vitalidade dos recém-nascidos esteve preservada na totalidade dos casos, pois o Apgar no primeiro e quinto minutos esteve acima de 7, em todos os casos. Embora o Apgar seja um índice limitado para avaliação das condições neonatais, é importante constatar que nenhum bebê do estudo teve asfixia ao nascer.

Neste estudo, foi investigada a queixa de ardor ao urinar, considerado como desconforto que poderia estar associado à presença de trauma perineal. No entanto, os resultados foram semelhantes no grupo com períneo íntegro $(\mathrm{p}=0,229)$. Vale destacar que pequenas soluções de continuidade da mucosa vulvo-vaginal, que não requerem sutura, podem provocar esse sintoma, nas mulheres incluídas no grupo sem laceração.

Pesquisa realizada com 100 puérperas que tiveram parto vaginal, verificou que a micção estava acompanhada de dor ou desconforto em $96,4 \%$ e $85,7 \%$ dos casos, no primeiro e terceiro dia de pós-parto, respectivamente ${ }^{(29)}$.

Estudo citado anteriormente ${ }^{(20)}$, caracterizou a dor referida por mulheres em relação à severidade dos traumatismos do períneo, observando que a dor no primeiro, segundo e décimo dias e aos três meses após o parto, assim como a frequiência do uso de analgésicos ocorria em ordem crescente, respectivamente, no períneo íntegro, laceração espontânea, episiotomia e lacerações de terceiro e quarto graus.

Deve-se considerar que conhecer os fatores relacionados à ocorrência de lacerações perineais no parto normal pode representar uma contribuição para a prevenção desse tipo de trauma e da morbidade que acompanha essa intercorrência.

\section{CONCLUSÃO}

Conforme mostram os resultados, os fatores constitucionais e assistenciais da parturiente, recém-nascido e parto em si - altura perineal, duração do período expulsivo, tipo 
de puxo, variedade de posição no desprendimento cefálico, circular de cordão umbilical e peso do recém-nascido - não interferiram na ocorrência ou grau de laceração perineal no

\section{REFERÊNCIAS}

(1) Organização Mundial da Saúde (OMS). Assistência ao parto normal: um guia prático. Brasília: OPAS/USAID; 1996. [OMS/ SRF/MSM/96.24].

(2) Brasil. Ministério da Saúde. Secretaria de Políticas de Saúde. Área Técnica de Saúde da Mulher. Parto, aborto e puérperio: assistência humanizada à mulher. Brasília; 2001.

(3) Cunningham FG, ManDonald PC, Gant NF, Leveno KJ, Gilstrap LC, Hankins GDV, et al. Williams obstetrícia. $18^{\mathrm{a}}$ ed. Rio de Janeiro: Guanabara Koogan; 2000. Conduta no trabalho de parto e parto normal; cap. 16, p. 309-25.

(4) Graham ID. Episiotomy: challenging obstetric interventions. London: Blackwell Science; 1997. The americam crusade for prophylatic episiotomy; chap. 3, p. 33-41.

(5) Diniz SG. Só um corte a mais? [editorial]. Notas sobre nascimento e parto. Genp. 1998;3(6):1.

(6) Martins-Costa S, Ramos JGL, Brietzke E, Stuazinaki JV, Dias EC. Episiotomia: o que há de verdade neste tradicional procedimento? Femina. 2001;29(4):201-4.

(7) Carroli G, Belizan J. Episiotomy for vaginal birth. Cochrane Database Syst Rev. 2000;(2):CD000081.

(8) Gerrits DD, Brand R, Gravenhorst JB. The use of an episiotomy in relation to the professional education of the delivery attendent. Eur J Obstet Gynecol Reprod Biol. 1994;56(2):103-6.

(9) Tomasso G. Debemos seguir haciendo la episiotomía en forma rutinaria? Rev Obstet Gynecol Venezuela. 2002;62(2):115-21

(10) Basile ALO. Estudo randomizado controlado entre as posições de parto: litotômica e lateral esquerda [dissertação]. São Paulo: Escola Paulista de Medicina da UNIFESP; 2000.

(11) Labrecque M, Eason E, Daniels F, Ymayo MR, Bourget M. Episiotomia de rotina: evidências contra. Diag Trat. 2000;5(2):43-50.

(12) Harrison RF, Brennam M, Noth PM, Reed JV. Is routine episiotomy necessary? Br Med J. 1984;288(6435):1971-5.

(13) Silva SF. Sutura do trauma perineal no parto normal: estudo comparativo entre duas técnicas [dissertação]. São Paulo: Escola de Enfermagem da USP; 2002.

(14) Argentine Episiotomy Trial Collaborative Group. Routine vs selective episiotomy: a randomized controlled trial. Lancet. 1993;342:1517-8.

(15) Albers LL, Anderson D, Cragin L, Daniels SM, Hunter C, Sedler KD, et al. Factors related to perineal trauma in childbirth. J Nurse Midwifery. 1996;41(4):269-75

(16) Costa ASC. Proteção perineal e manejo do feto no parto normal: estudo comparativo entre duas técnicas [dissertação]. São Paulo: Escola de Enfermagem da USP; 2002. parto normal. Igualmente, o ardor ao urinar não pôde ser atribuído ao trauma perineal.

(17) Silva FMB, Oliveira SMJV, Koiffman MD, Osava, RH. Caracterização de parturientes atendidas em um centro de parto normal. In: Anais do $2^{\circ}$ Encontro Internacional de Pesquisa em Enfermagem: trajetória espaço-temporal da pesquisa; 2002 out. 28-31 Águas de Lindóia. [CD-ROM] São Paulo: EEUSP; 2002 .

(18) Klein MC, Janssen PA, MacWilliam L, Kaczorowski J, Johnson B. Determinants of vaginal-perineal integrity and pelvic floor functioning in childbirth. Am J Obstet Gynecol. 1997; 176:403-10.

(19) Sampselle CM, Hines S. Spontaneous pushing during birth relationship to perineal outcomes. J Nurse Midwifery. 1999; 44(1):36-9.

(20) Klein MC, Gauthier RJ, Robbins JM, Kaczorowski J, Jorgesein $\mathrm{SH}$, Franco ED, et al. Relationshipof episiotomy to perineal trauma and morbidity, sexual dysfunction, and pelvic floor relaxation. Am J Obstet Gynecol. 1994;171(3):591-8.

(21) Howard D, Davies PS, DeLancey JO, Small Y. Differences in perineal lacerations in black and white primiparas. Obstet Gynecol. 2000;96(4):622-4.

(22) Goldberg J, Hyslop T, Tolosa JE, Sultana C. Racial differences in severe perineal lacerations after vaginal delivery. Am J Obstet Gynecol. 2003;188:1063-7.

(23) Shipman MK, Boniface DR, Tefft ME, McCloghry F. Antenatal perineal massage and subsequent perineal outcomes: a randomised controlled trial. Br J Obstet Gynaecol. 1997; 104:787-91.

(24) Abeche AM, Teruchkin B, Leite CSM, Mucenic M. Episiotomia: reavaliação de um procedimento clássico. Femina. 1994;22(6/9):436-40.

(25) Jones M, Larson E. Length of normal labor in women of hispanic origin. J Midwifery Womens Health. 2003:48(1): $2-9$.

(26) Roberts JE, Goldstein SA, Gruener JS, Maggio M, MendezBauer C. A descriptive analysis of involuntary bearing-down efforts during the expulsive phase of labor. JOGNN. 1987;16(1):48-55.

(27) Enkin MW, Keirse MJNC, Reefrew MJ, Neilson JP. A guide to effective care in pregnancy and childbirth. $2^{\text {nd }}$ ed. Oxford: Oxford University Press; 1999. The second stage of labour; chap. 32, p. 226-35.

(28) Lydon-Rochelle MT, Albers L, Teaf D. Perineal outcomes and nurse-midwifery management. J Nurse Midwifery. 1995; 40(1):13-8.

(29) Alexandre CW. Prevalência, características e impacto da dor no cotidiano da puérpera [dissertação]. São Paulo: Escola de Enfermagem da USP; 2002. 